\title{
Immune responses to macroparasites are sensitive to the interaction between genetic growth potential and protein nutrition in mice
}

\author{
J. C. Coltherd ${ }^{1}$, S. Babayan ${ }^{2}$, L. Bünger ${ }^{3}$, I. Kyriazakis ${ }^{1,4}$, J. E. Allen ${ }^{2}$ and J. G. M. Houdijk ${ }^{1}$ \\ ${ }^{1}$ Animal Health, SAC, Edinburgh EH9 3JG, UK, ${ }^{2} I I I R$, Ashworth Laboratories, Edinburgh EH9 3JT, UK, \\ ${ }^{3}$ Animal Breeding and Genetics Team, SAC, Edinburgh EH9 3JG, UK and ${ }^{4}$ Veterinary Faculty, University of Thessaly, \\ 43100 Karditsa, Greece
}

\begin{abstract}
Animals selected narrowly for increased production traits may be penalised more from pathogen challenges than their unselected counterparts. These genetic correlations between production and immune traits could be based on changes in the allocation of scarce resources to immune functions. This possibility is addressed in mice that have been divergently selected over thirty-eight generations for high $(\mathrm{ROH})$ and low $(\mathrm{ROL})$ body weight at $42 \mathrm{~d}$ of age ${ }^{(1)}$. Infection with a gastrointestinal parasite in ROH mice results in a greater penalty on resilience (body weight and food intake) and resistance (parasite egg numbers and worm burden) than in ROL mice ${ }^{(2)}$. This penalty on resilience is sensitive to protein nutrition, but the expected sensitivity of resistance to protein nutrition ${ }^{(3)}$ is not significant. The aim of the current study was to assess host immune responses that may underlie interactive effects of genetic potential and protein nutrition on resistance to parasites.

In a $2 \times 2 \times 2$ design, individually-housed male ROL and ROH mice were fed ad libitum diets with either 40 (LP) or 230 (HP) g crude protein $(\mathrm{N} \times 6.25) / \mathrm{kg}$ and either sham infected or infected with Heligmosomoides bakeri larvae at $32 \mathrm{~d}$ of age $(n 6)$. Feed intake and body weight were assessed twice weekly for $28 \mathrm{~d}$ when worm burdens, cytokine and antibody levels were analysed. Cytokine production was analysed using GLMM with a gamma distribution while all other data parameters were analysed using ANOVA.

Infection reduced body-weight gain in ROH-HP mice to a smaller extent than in ROH-LP mice (HP $1.71 \mathrm{~g} v$. LP $2.72 \mathrm{~g}$, SED 1.5 ; $P=0.035)$. In addition, ROH-HP mice had lower worm burdens than ROH-LP mice $(P=0.077$; Fig. 1$)$. These effects were not seen in ROL mice. Infection with $H$. bakeri increased levels of IL-13 $(P=0.009)$ and IL-5 $(P=0.001)$ in all mice. Across genotype, HP mice had higher levels of IL-5 $(P=0.021)$ and lower levels of IL-6 $(P=0.008)$ than LP-mice (Fig. 2). In ROL mice infection increased levels of interferon- $\gamma(\mathrm{IFN}-\gamma)(P=0.026)$ and decreased levels of IL-4 $(P=0.003)$. Infection-increased IgG1 in both genetic lines but to a lesser extent in the ROL mice $(P=0.027)$.
\end{abstract}

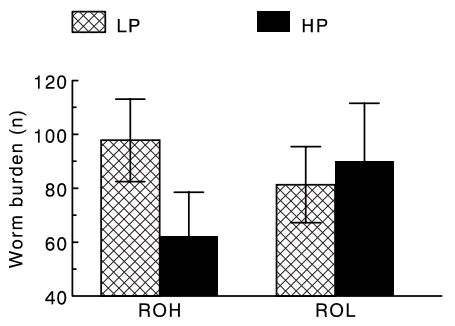

Fig. 1. Worm burdens on LP and HP diet.

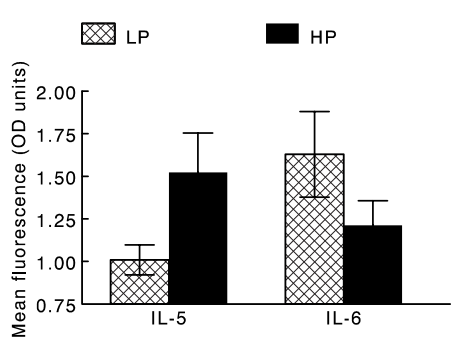

Fig. 2. IL-5 and IL-6 on LP and HP diet.

During H. bakeri infection, alongside a generalised increase in T-helper (Th) 2 cytokines (IL-5 and IL-13), selected immune responses were found to be sensitive to protein nutrition (IL-5 and IL-6). It was also found that ROL mice produced a more inflammatory response to infection, with increased levels of IL- 6 and IFN- $\gamma$ compared with ROH mice. These observations suggest that narrow selection for differences in body weight may fundamentally alter host immune responses towards gastrointestinal parasites.

1. Bünger L, Laidlaw A, Bulfield G et al. (2001) Mamm Genome 12, 678-686.

2. Coltherd JC, Bünger L, Kyriazakis I et al. (2009) Parasitology 136, 1043-1055.

3. Boulay M, Scott ME, Conly SL et al. (1998) Parasitology 116, 449-462. 